\title{
JUNIOR NATURALISTS' SECTION IN BLUE JAY, 1954 - 1972
}

MARY I. HOUSTON, 863 University Drive, Saskatoon SK S7N 0J8

In the 1953 fall issue of Blue Jay, Elizabeth Hubbard of Grenfell proposed a contest for students to encourage their interest in nature observation. In 1954 the first "Nature's School house-Boys' and Girls' Section" appeared. First, second and third prizes for the best contributions were given following each issue. Donated by individual society members, prizes consisted initially of a choice of any Peterson's Field Guide or Wherry's Flower Guide. The grand prize for the first seven issues, a $\$ 50$ camera donated by Dick Bird of Regina, was won by Wilma Aim for her article about the muskrats on her farm near Bredenbury. ${ }^{1}$ These were valuable prizes for that time. Later winners had an added choice of Photography for Teenagers, a subscription to Canadian
Nature, or a three-year subscription to Blue Jay. By 1963 , the prize was simply a one-year subscription to Blue Jay. Prizes were last given in 1965.

In 1957, Joyce Dew (Figure 1) from the Saskatchewan Museum of Natural History became the editor of the youth section. In 1961, the name of the section was changed to Junior Naturalists. Joyce married Adam Deutscher in 1962 and left the employ of the museum in 1963 to become a high school art teacher, but continued as an Associate Editor in charge of youth submissions until 1970. In 1971 and 1972, junior contributions continued in diminishing numbers, but were no longer in a separate section; the age of the some contributors was still provided.

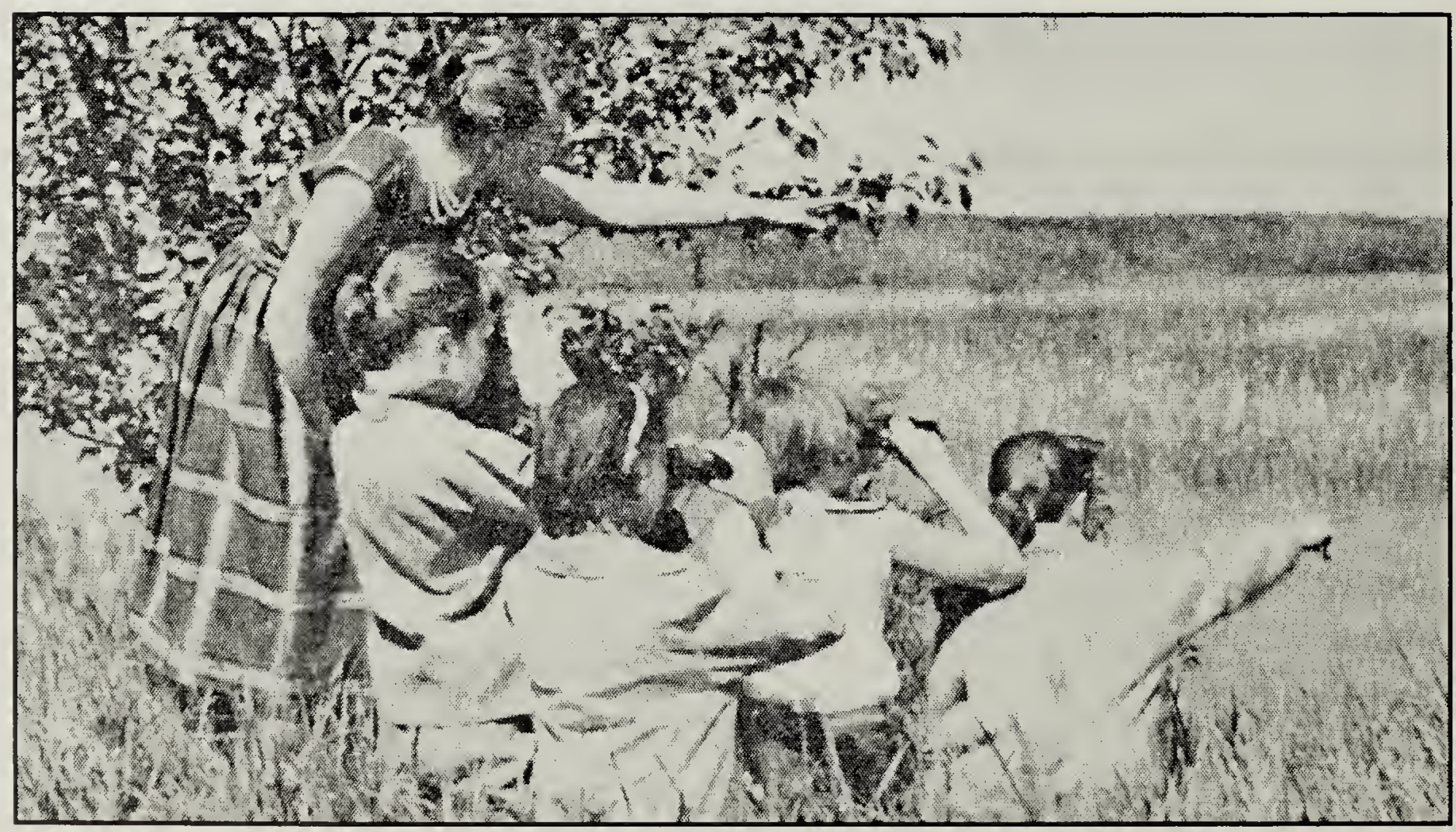

Children birdwatching with Joyce Dew in Regina Waterfowl Park Fred G. Bard 
Between 1954 and 1972, over 500 short articles, drawings, poems and letters were contributed by 380 young people from 165 locations. Most contributed only one item, but over 50 contributed two or more. The title, "honour naturalist" for "juniors who contribute more or less regularly" ${ }^{2}$ was given to two people: Bohdan Pylypec of Yellow Creek who had 18 items printed, and Brian Irving of Kelvington with 17. Rosemary Nemeth of Yellow Creek had 13.

Over the years, the contributors ranged from grade 1 students, aged six years, to high school students and two recent high school graduates. The

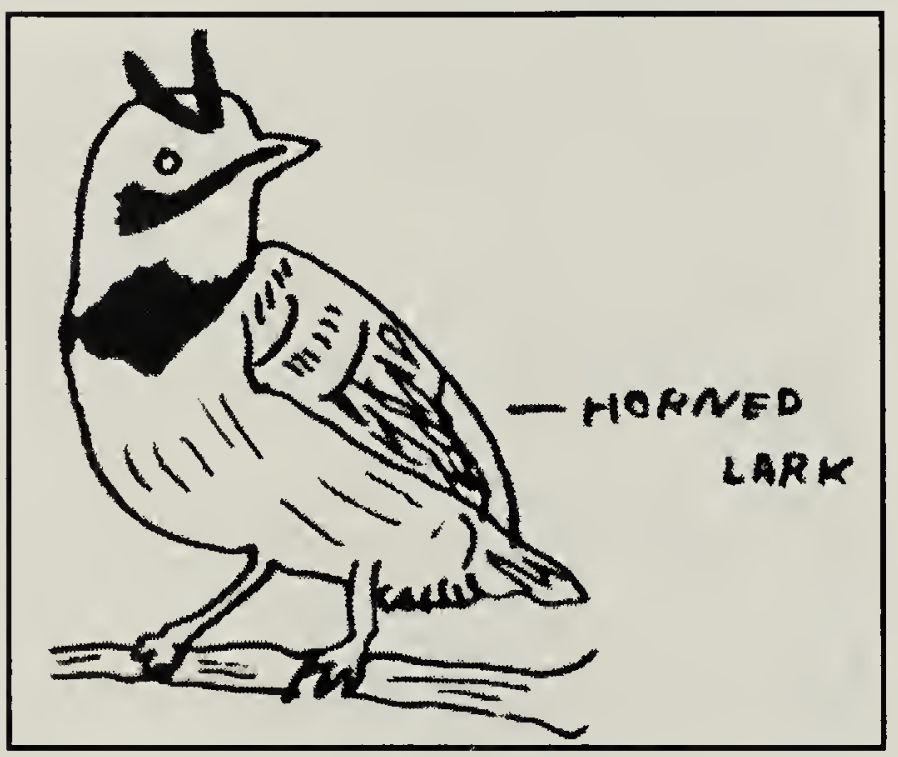

Drawing by Dan Pawlivsky, age 10, Square Hill, Sask.

most common ages $(77 \%)$ for initial submissions were between 11 and 14 , usually in grades 5 to 7 . Interest came from a wide geographic area, indicating the extent of Blue Jay subscriptions. Several hundred schools benefitted from bulk purchases by the regional school boards. Contributions came from 325 Saskatchewan students from 128 localities (including now-defunct post offices such as Adams and Hearne; rural schools qualified as separate localities prior to universal ownership of cars and trucks), 26 from Manitoba and 17 from Alberta, as well as from outside the Canadian prairies: British Columbia (3), Ontario (3), NorthWest Territories (2), Missouri (1), Montana (1), Wisconsin (1) and two items, one a prize-winner, from a lad in London, England. Yellow Creek school, with Bryan Isinger as teacher, submitted contributions from 46 juniors. (See the list at the end of this article).

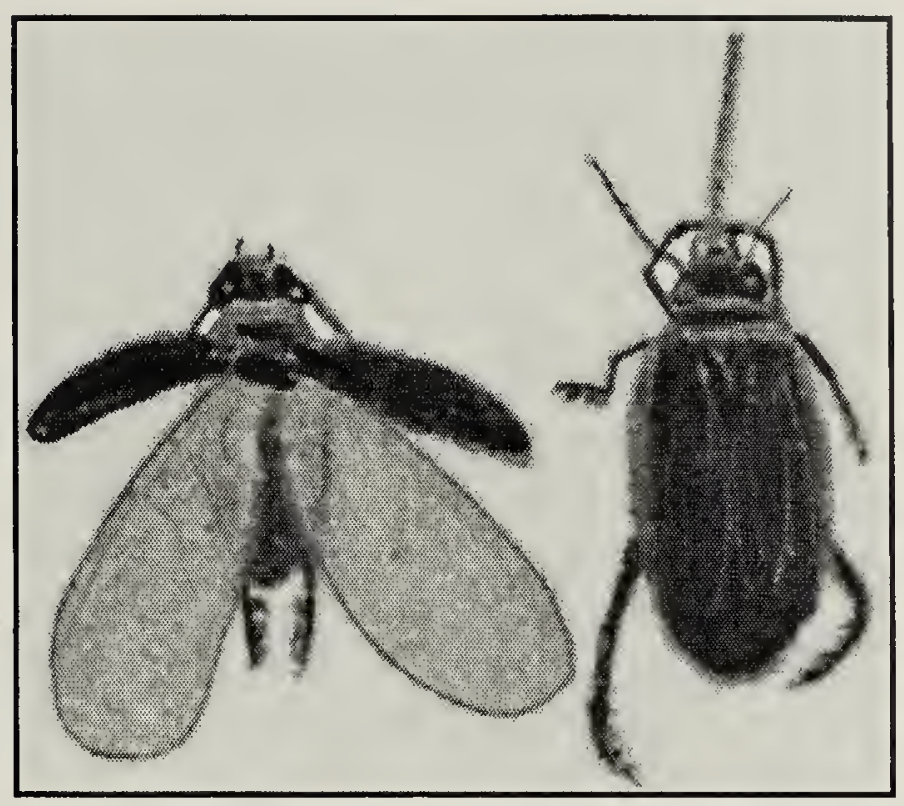

Koko, the Predacious Diving Beetle, by June Martinsen

Schools were stimulated to form nature clubs. These included clubs at Simpson School (run by Miss Pauline Summers) in Yorkton, Lonsdale School near Loon Lake, Omand School near Kinistino, Kitzman School near Rhein, the coincidentally-named Blue Jay School near Carrot River, Thunderbird School near Benito, Manitoba, and the "Seagull club" at Meath Park. Each of these clubs sent in accounts of their projects and nature hikes. There was a Junior Exhibit on display at the Saskatchewan Museum of Natural History in 1962. The Brandon Juniors contributed an annual report on bird box success from 1963 to 1976 , the final two years submitted by Mrs. Lane after Jack Lane's death.

As one would expect, some youngsters wrote about topics such as a special pet, important to them but of little significance in a wider sense. 
Others reported natural history observations of lasting value: the first sighting of a Steller's Jay in the Saskatoon area on October 29, 1961; a Ruffed Grouse and a Mallard nest only two feet apart near Kamsack on May 3, 1958; ${ }^{4}$ the first white phase Gyrfalcon in the Yorkton-Duck Mountain area on March 29, 1959, ${ }^{5}$ and a Whip-poor-will seen near Hudson Bay in $1965 .^{6}$

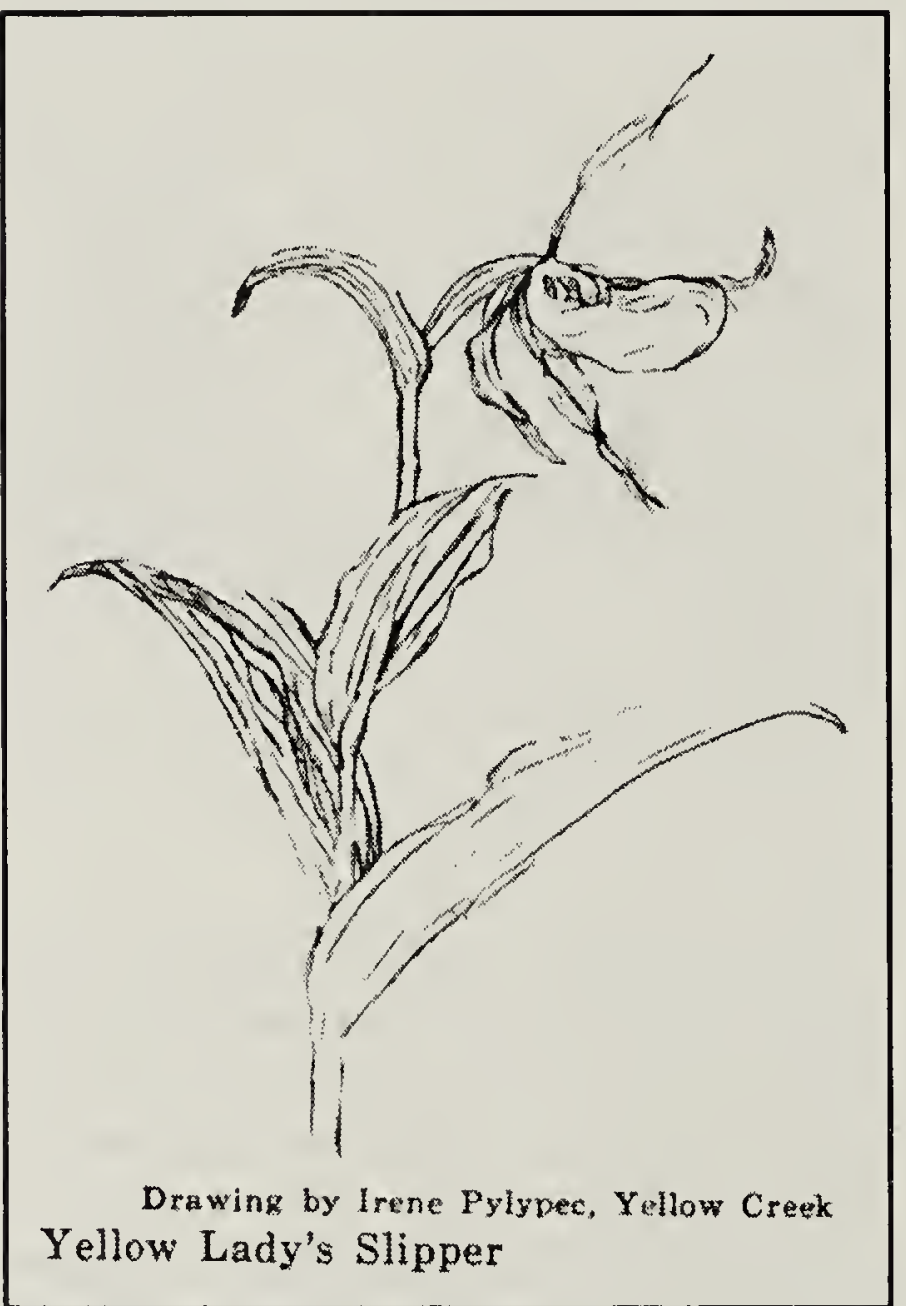

Names of students who, from 1954 - 1970, had articles published in the Boys' and Girls' Section and the Junior Naturalists section, or whose unpublished articles received mention, are listed in the following Appendix. Locations are listed alphabetically within regions, which are listed in order of the number of people submitting from each. An asterisk beside a name indicates those students who also participated in Saskatchewan CBCs.

1. AIM, W. Furry Boarders. Blue Jay 12(3): 15-17.

2. DEW, J. 1965. Comments and prize winners. Blue Jay 23:102

3. GOLLOP, M. 1962. Surprise. Blue Jay 20:46.

4. JMAEFF, J. H. 1958. Peaceful neighbors. Blue Jay 16:128.

5. JMAEFF, J. H. 1959. A gyrfalcon observation. Blue Jay 17:77.

6. MALUTA, B. 1966. Whip-poor-will at Hudson Bay. Blue Jay 24:94.
A second article in preparation will tell about earlier and concurrent articles by Saskatchewan juniors, outside of the Junior Naturalists section. It will assess participation by juniors in other activities such as Christmas Bird Counts, Co-operative Spring Migration Surveys, the Prairie Nest Records Scheme, bluebird house trails, and the American Ornithologists' Union meeting in Regina in 1959.

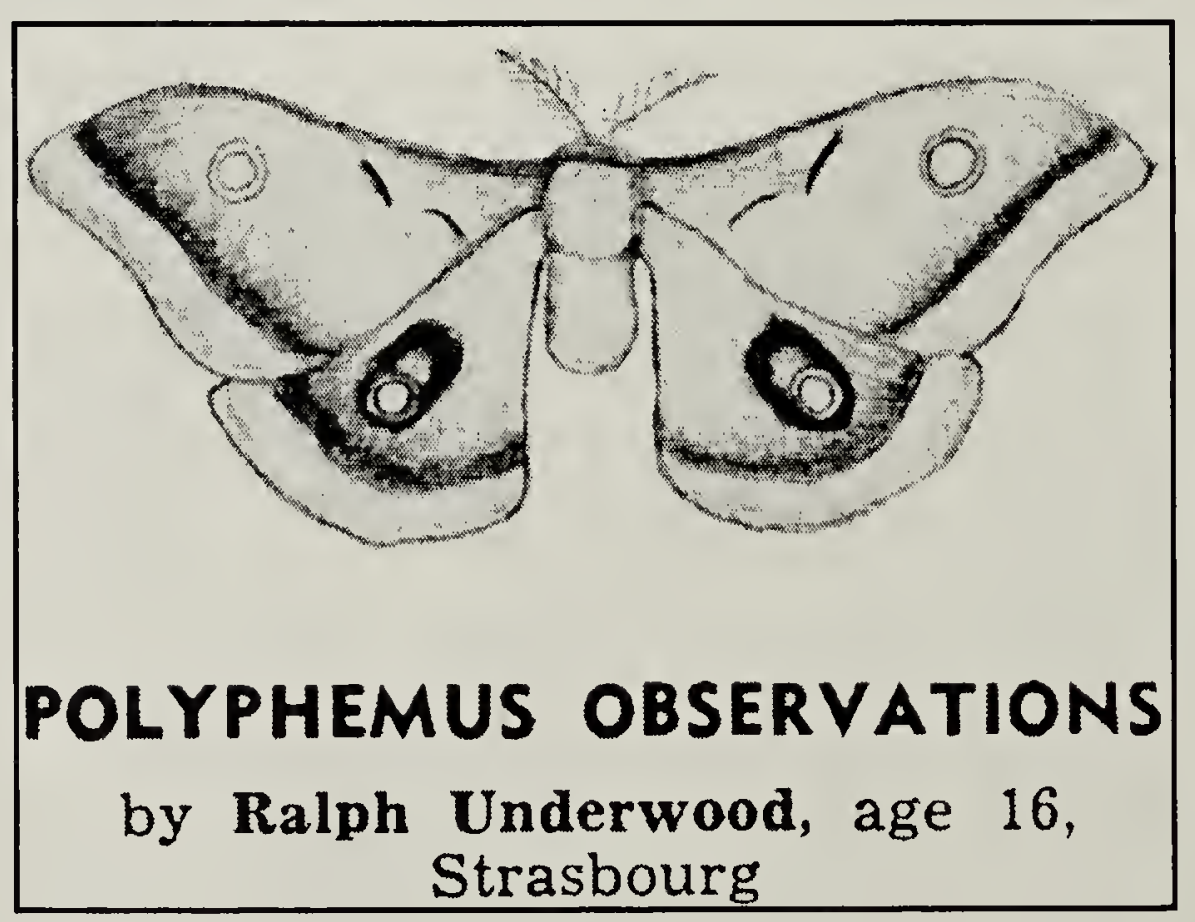




\section{APPENDIX}

\section{SASKATCHEWAN (325)}

Abernethy: Bryan Lyster; Adams: Kenneth Howland; South Allan School, Allan: Alan Boon; Anglia: Alice Bawron; Everton School, Archerwill: Fay Johnsen; Armley: Jim Breadner; Arran: Doreen Maksymchuk, Harry Wolf; Barthel: Herbert Mimrichter; Beechy: Alfred Jones; Bladworth: Sam Beckie*, Linda Edwards; Blaine Lake: Jean Kulick, Lyn Lamers, June Lamers; Bredenbury: Wilma Aim, Kathleen Gunnarsson; CutArm School, Bredenbury: George Mess, Sheila Mess, Joyanne Polson, Audrey Roberts; Broadview: Audrey Carpenter, David Chaskavich*, Charles Feathers, Brian James, Cheryl Jensen, Alfred Lange, Don Weidl*, Doreen Wilson; Buffalo Narrows: Gerald Laliberte; Calder: Eunice Gawdun, Verna Horaska, Rachel Niniowski, Colleen Schab, Lawrence Scraba; Carmichael: Roberta Forsaith;

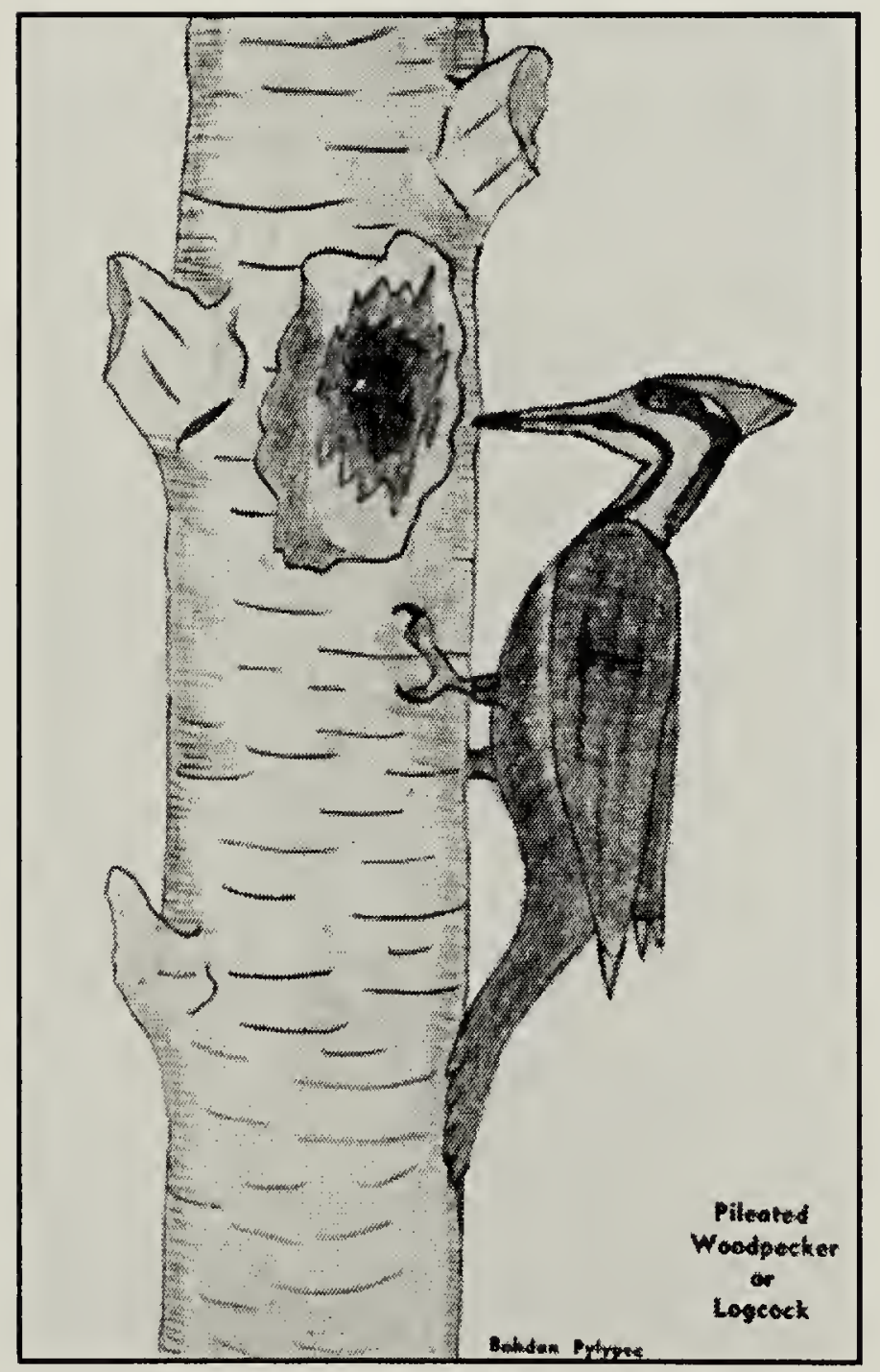

Carnduff: Richard Hogg; Carrot River: Paul Fowler, Ronald Nickel; Churchbridge: Herbert Hlady; Clouston: Edwin Kuchn; Cochin: Betty Ternier; Coderre: Donna Anderson, Joan Anderson; Dalmeny: Richard Loewen; Dollard: Marvin Morvik; Dore Lake: Maxine Johnson; Dubuc: George Chopping*; Dundurn: Harry Harder*; Elrose: Garnet Ward; Endeavour: Gordon Sawchuk; Englefeld: Beverly Herriges; Estevan: Sheila Carlson, Ross Lein*; Evesham: Lynn Sumner; Flaxcombe: Marion Clark; Fort-a-laCorne: Ernest Sauve; Gerald: George Hruska, Merle Remus; Goodwater: Edward Hennon; Grand Coulee: Murray Rowan; Grenfell: Jim Elliott, Elizabeth Hubbard, Linden Hubbard; Hagen: Lorne Graupe, Ernest Skaar; Hanley: Glen Hass; Hearne: Frederick Gase; Holbein: Susan Pegg; Hudson Bay: Pat Earnshaw, Brian Maluta; Indian Head: Denise Beaulieu*, Peter Carton, Janet Gray*, Sharon Hubbs, Tim Keslering, Heather McLaughlin, Margaret McLaughlin, David Mounteney, Brian Scott, Kathy Skinner, Jackie Willerth; Marguerite School, Indian Head: Annette Francaise, Claire Francaise, Marcel Francaise, Lynne Simpson; Invermay: Myles Ferrie, Ed Shepherd; Kamsack: Judy Dubasov, Jacob Jmaeff*, Debbie Niniowski, Philip Olshewski; White Rose School, Kegworth: Helen Furtan, Elenor Konechny; Kelliher: Wayne Bernakevitch; Kelvington: Brian Irving * Jack Lowndes, William Saban, Dianne Sloan*; Kendal: Joe Michel; Killaly: Johnny Kerelchuk; Kindersley: Glen Fox*, Lane Harris*; Omand School, Kinistino: Beverly Beaulieu, Gary Beaulieu, Linda Beaulieu, Brenda Gudelot, Gail Gudelot, Gary Hills, Marlene Hills, Barry Warbis; Hazel Valley School, Kipling: Douglas Cross, Brad Lawrence, Lionel Meszaros; Lac la Ronge: Lynn Ernst; Lady Lake: Donald Buckle*; Leross: Sylvia Bordass, Dale Brennan; 


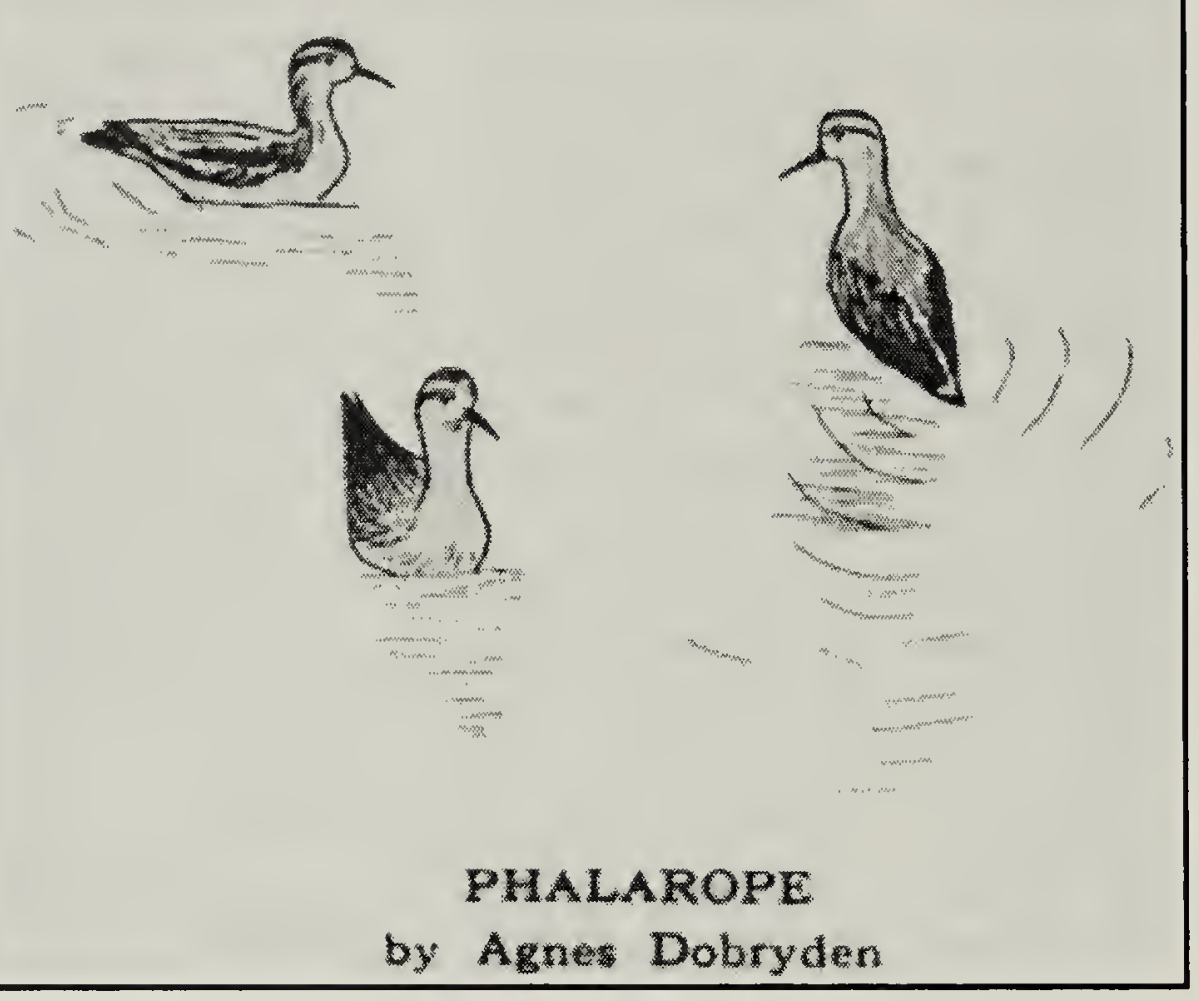

Keith Harper; Porcupine Plain: Gladys Beveridge; Prelate: Ronald Gizin, Nick Stebanuk; Prince Albert: Bernice Capusten, Donald Karasiuk*, Jimmy Zulkoskey; Radisson: Bryan Rothenburger*; Redvers: Sheila Purvis; Regina: Richard Albright, Trevor Apperley, Ronald Austin*, Bob Clements, Debra Doering, Bill Fleming*, Jan de Groot, Ruth Faith Herman, Erica Hoffman, Doreen Jasper, Harriet Jowsey*, Bob

Lloydminster: Jean Gould; Lorlie: Margaret Schick; Lucky Lake: John Bayless, Nicky Boon; MacNutt: Laverne Wendell; Madison: Karen Knight, Vicki Knight, Elizabeth McManus; Mankota: Darlene Svinger; Marieval: Margaret Bawden; Maryfield: Eileen Adair, Mildred Boon, Morley Bullock, Dale Flynn, Brenda Matte, Murray Moore, Laurie Robinson; Marysburg: Gregory Schulte; Melfort: Ian Kinnan; Mennon: Bob Buhr*; Montmartre: Gerald Wilke; Moose Jaw: Carol Andrews, Douglas Ewart ${ }^{\star}$, Mary Gilles, Moray Lewis*, John Rhodes, Michael Rhodes*, Jack Zess*; Moosomin: Roberta Smith; Moreland: James Liggett; Muscow: Maureen Cyr, Lawrence Herperger; Naicam: Ron Jensen*, Murray Thompson; Nipawin: Sharon Evans, Anne Matthews*, David Riome*; Oakshela: Lyndon Reeve; Edgewood School, Oakshela: Carolina Kotulski; Ogema: Bob Turner; Okla: Lawrence Lamber; Oxbow: Robert Galloway; Parkman: Carolyn Johnson, Patricia Johnson, Rita Mursell, Joyce Smith, Dorothy Taylor; Peebles: Douglas MacFarlane; Pense: Beverly Esplen, Bonnie Frew; Star Butte School, Piapot: Glenn Fairweather, Dixie Hobbs, Liane Nielsen; Pleasantdale:
Kerr, Elizabeth Lane, June Martinsen, Wayne Miller, Sally Moss*, Lorrel Nero, Tamara Nero, Joey Purdy, Rick Sanderson*, Daryl Schaffer, Alex Steele, Peter Tsakires, Alan Wade*, Elizabeth Wagner*, Sherry Wallace, Shawn Wallace, Peter Wayte, Jack Wessel; Rhein: Karon Brooks, Marvin Hrynowetsky, Marjorie Wardlaw; Rocanville: Shirley Anderson; Rokeby: Gerald Kreba, Garry Thompson; Rosthern: Edward Andres; Saltcoats: Janice Bradshaw, David Farrell, Bill

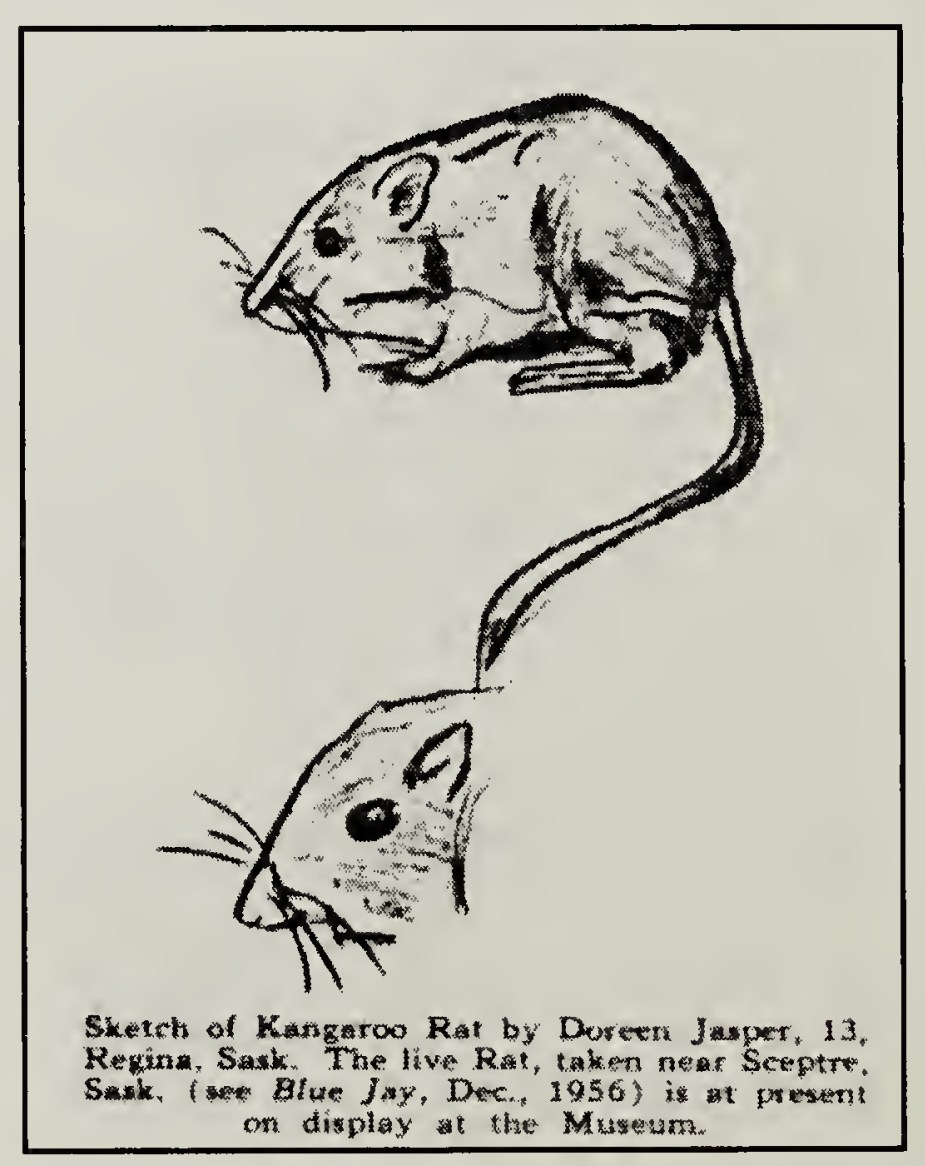


Horseman*, Ronnie Kirkham; Chatsworth School, Saltcoats, Evelyn Mess, Lynne Maddaford; Saskatoon: Mark Abley*, Gary Adams, Mike Bantjes*, Joan Besant*, Barbara Binnie, Michael Gollop*, David Houston*, Ellen Nykyforuk, Tom Nykyforuk, Joanne Waite, Carol Van Dyck; Semans: Jane Richardson; Shaunavon: Douglas Young; Shell Lake: Joel Loseth; Shellbrook: Debra Hamilton; Somme: Don Hooper, Ron Hooper; Springside: Doreen Kovalyk, Stanley Pawliw, Dennis Simair; Square Hill: Dan Pawlivsky; Stornoway: Joyce Klingman, Grant Mehling; Strasbourg: Lois Everett, Kenneth Underwood, Ralph Underwood; Sturgis: Valerie Johnson; Swift Current: Mary Whyte; Tarnopol: Theodore Chytyk, Arlene Swicheniuk, Gerald Swicheniuk; Tisdale: Peter Skichan, Kenny Stirrest, Kenny Tompkins; Tonkin: Nelson Cherry, Colleen Farrell, Sharon Pearce; Torch River: Brian Evans, Dwight Hayes, Kathleen O'Drowski [a later submission came from Prince George, BC]; Tregarva: Kenneth

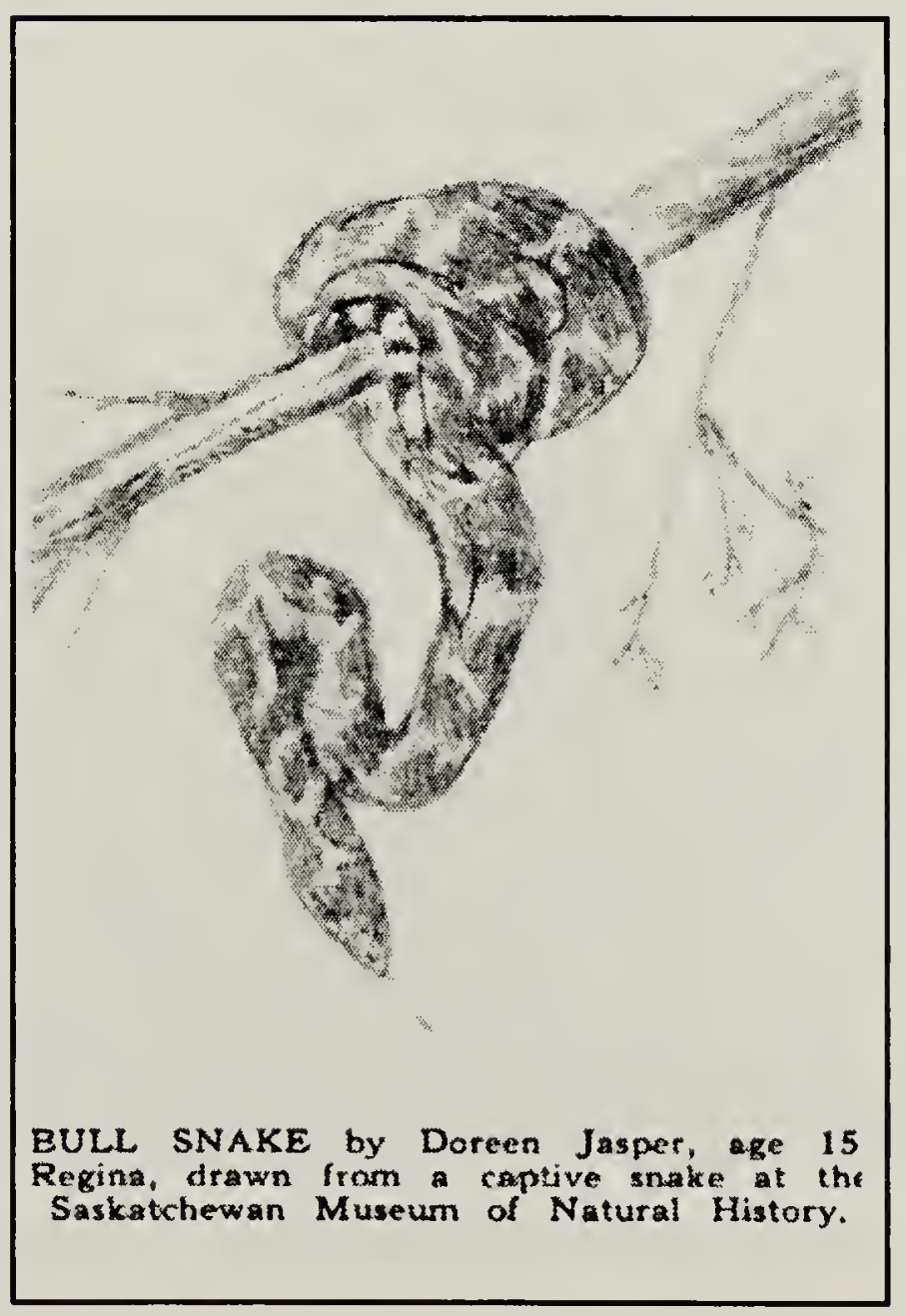

Dickson; Trossachs:Gail Bernard, Corinne Goodwin; Unity: Mary Gillies; Uranium City: Pat Kerr; Usherville: Gordon Skuce; Valley Centre: Wayne Renaud*; Waitville: Lyall Hunter; Whitewood: Nicholas Shurvin; Wilkie: Phillip Keller; Windthorst: Gayle Dayman; Yellow Creek: Sherry Chubak, Delmer Dutka, Glen Flemming, Debby Gingara, Stella Holinaty, Colette Isinger, Robert Kotyk, Eugenia Koval, Josephine Kruppi, Donald Krytzak, Ellen Kuz, Byron Lane, Audrey Lebedowich, Evelyn Lypchuk, Dennis Matkowsky, Donna Matkowsky, Darwin Mazur, Derwent Mazur*, Gaylene Mazur*, Kenneth Mazur, John Mukli, Elsie Nemeth, Linda Nemeth, Rosemary Nemeth*, Bobby Oleksyn, Gary Oleksyn, Oli Oleksyn, Glen Orenchuk, Gordon Orenchuk, Kenneth Orenchuk, Rita Ozimirski, Joan Popiel, Irene Pylypec*, Bohdan Pylypec*, Iris Sheremata, Marie Sheremata, Colleen Shewchuk, Deborah Shewchuk, Allen Simon, Iris Simon, Linda Simon, Idelle Swickiniuk, Elizabeth Toth, Glen Wilchynski, Don Wojcichowsky, Audrey Zip; Rhona Lake School, Yellow Creek: Marion Kruppi; Yorkton: Margaret Houston*, Roxby Hughes*, Eugene Stauffer, Darcy Wershler*; Young: Marleen Christensen.

\section{MANITOBA (26)}

Benito: Lydia Koziol, Mary Koziol, John Rezansaff; Brandon: Randy Bauman, Vern Bauman, Brian Cutforth, Jack Endall, D. Knock, Wayne Miller, John Nicol, Richard Patterson, Bruce Peters, David Plews, Billie Pugh, David Randall, Dale Robinson, Gordon Rourke, Les Rourke, Doug Wong; Cartwright: Glen Watts; Elma: Gloria Tommila; Russell: Ron Klimack; Sanford: Agnes Dobryden; Shoal Lake: Derrick Edmundsen; St. James: Dawn Brodie; Winnipeg: Jonathan Davis. 


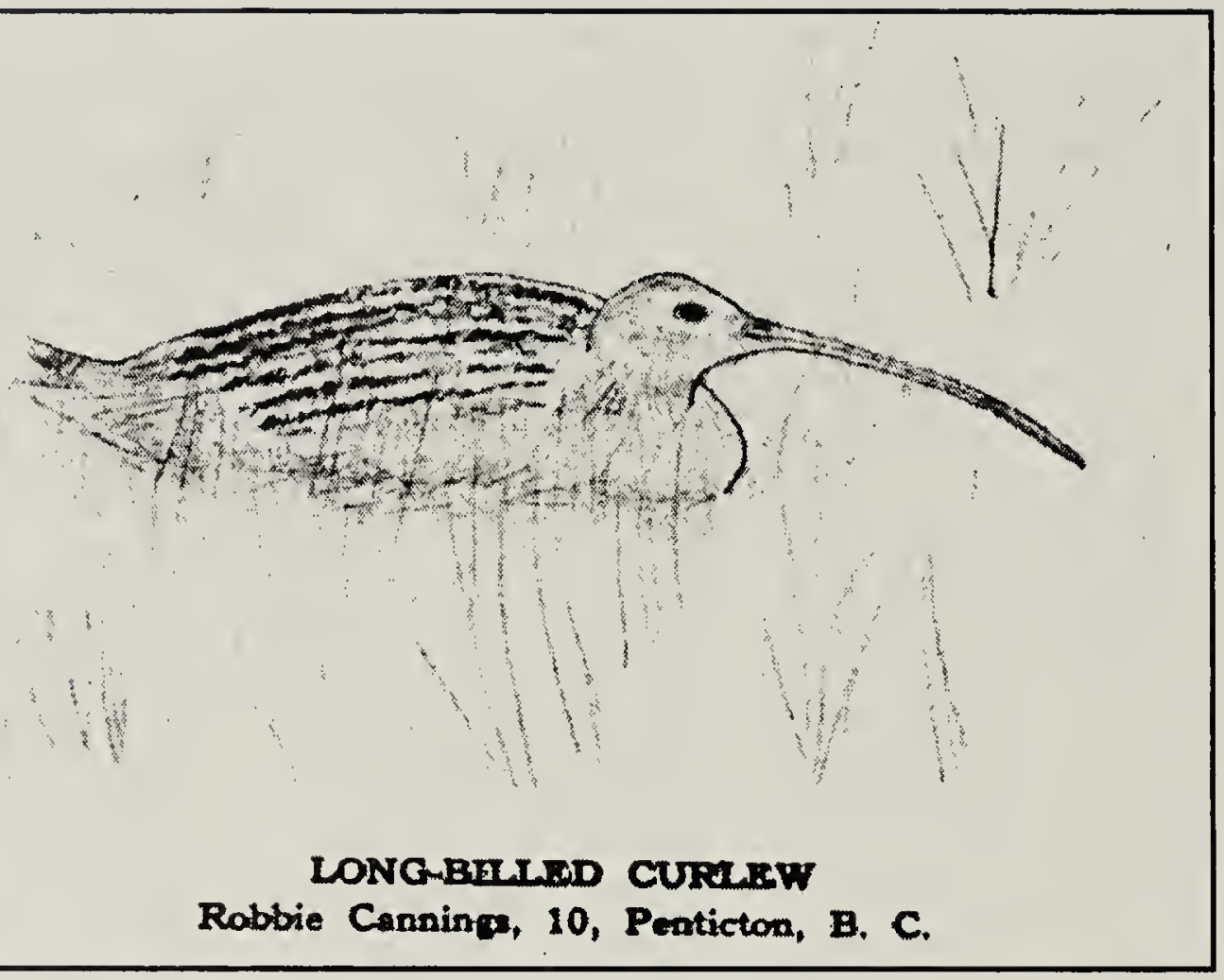

BRITISH

COLUMBIA (3)

Penticton:

Bette

Cannings,

Robbie

Cannings;

Vernon: Robin

Tamasi.

ONTARIO (3)

Camp

Robinson:

Nancy Reid;

Islington: Len

Nash;

Sudbury: Pat

Seifert.

\section{ALBERTA (17)}

Botha: Jack Dayman; Brownfield: Garry [no surname]; Calgary: Kevin van Tighem; Carvel: Henry Savard; Chauvin: Stella Ronaghan; Codesa: Duane Hillaby; Edmonton: David Hobson; Erskine: Evelyn Lohr; Fleet: Stanley Checkel; Fort Vermilion: Dan Ferguson; High River: Larry Rasmussen; Irma: May Ronaghan; Neutral Hills: Wanda Tennant; Onoway: Keith Turnbull; Thorsby: Bill Grasnick; Two Hills: Bill Greschuk; Vermilion: John Evans.

NORTH WEST TERRITORIES (2)

Fort Smith: Tom Bryant; Nahanni Butte: Mickey Kraus;

MISSOURI (1)

Columbia: Daniel Greenlaw.

MONTANA (1)

Glasgow: Diana Hartley.

WISCONSIN (1)

Laurie Thompson.

ENGLAND (1)

London: Keith Winstone

Cover photo story:

\section{YOUNG CEDAR WAXWINGS}

by William P. Saban, age 14, Kelvington

Last summer a pair of Cedar Waxwings made a nest in our caraganas near the house and another some distance away. Again this summer I found a waxwing nest containing four young ones. Three of the four seemed in good condition except the one which hatched later and appeared to be sleeping most of the time. In a few days the youngest one had grown as big as the other three.

I came upon this nest August the ninth and I guessed the young were two weeks old. On August twelfth all four had taken flight. P. Saban.

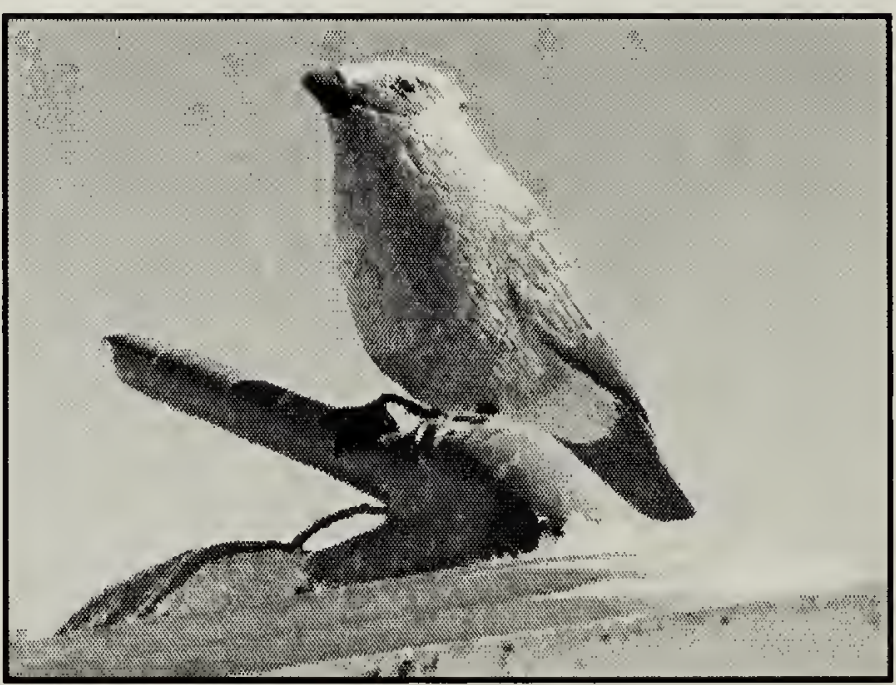

A recent waxwing carving by William 